\title{
Artrópodes em Ninhos de Columba livia Gmelin, 1789 (Aves, Columbidae) em Área Urbana de Manaus, Amazonas, Brasil.
}

\author{
Guilherme Maerschner Ogawa
}

Instituto Nacional de Pesquisas da Amazônia (INPA), Núcleo de Pesquisas em Ciências Humanas e Sociais. Laboratório de Etnoepidemiologia, e-mail do autor: guilelme@hotmail.com.

EntomoBrasilis 1(3): 69-72 (2008)

Resumo. Foi realizado um estudo sobre artrópodes que vivem em ninhos de Columba livia Gmelin 1789, ave conhecida como pombo urbano e que nidifica em construções humanas, com o intuito de inventariar as espécies de artrópodes associadas. Foram coletados 14 ninhos de $C$. livia em nove bairros da área urbana de Manaus os quais foram levados ao laboratótio e colocados em um extrator do tipo Berlese-Tulgren por 12 dias, para a captura dos artrópodes. Foram encontrados 10.323 artrópodes, pertencentes a 3 subfilos, 3 classes, 14 ordens e 33 famílias. Acari foi mais abundante com 7.879 indivíduos, sendo o gênero Caloglyphus Berlese, 1923, o mais representativo (75\%). A maioria das espécies encontradas é detritívora. A diversidade de artrópodes foi menor em Manaus se comparada à registrada em trabalhos da região Paleártica, provavelmente por $C$. livia ser uma espécie exótica na fauna Neotropical.

Palavras-chave: Artrópodes, entomologia urbana, fauna nidícola, pombo urbano.

\section{Arthropods in the Nests of Columba livia Gmelin 1789 (Aves, Columbidae) in the Urban Area of Manaus, Amazonas, Brazil.}

Abstract. The objective of this study was identify the arthropod fauna in nests of the urban pigeon, Columba livia Gmelin 1789 who build their nests in man-made buildings. Fourteen nests were collected in nine districts of the built up area of the city of Manaus. For twelve days, arthropods were extracted in a Berlese-Tulgren funnel. A total of 10.323 arthropods representing 3 subphyla, 3 classes, 14 orders and 33 families were detected. Acari was the most abundant group with 7879 individuals. Mites of the genus Caloglyphus Berlese, 1923, made up over $75 \%$ of all arthropods collected. Most of the arthropods extracted are detrivorous and apterous. The diversity found in Manaus was lower than that reported for samples collected in the Palearctic Region, probably because C. livia is exotic in the Neotropical fauna. The introduction of this pigeon can be responsible for a major contact between some arthropods and humans.

Key words: Arthropods, feral pigeon, nest fauna, urban entomology.

Cons olumba livia Gmelin, 1789, conhecida como pombo urbano, é uma ave sinantrópica da família Columbidae originária da Eurásia, de regiões que se estendem do norte da África até o norte da Europa e sudoeste da Ásia (Chiappe \& Dyke 2002). É encontrada em praticamente todas as grandes cidades do mundo. No Brasil, existem apenas populações urbanas da espécie, e acredita-se que esteja presente aqui desde o início da colonização européia. Seus ninhos são construídos com gravetos de 2 a $5 \mathrm{~mm}$ de diâmetro e 5 a $20 \mathrm{~cm}$ de comprimento, dispostos lado a lado e que podem ser colados com fezes e outras secreções da própria ave.

Ninhos de aves são microhabitats para diversos grupos de artrópodes (Hicks 1959; WlodarczyK 1963; New 1972; Aldrete 1988), que neles encontram abrigo e alimentos como os de origem vegetal, que dão formato e sustentação ao ninho, bem como restos de alimentos trazidos por adultos, penas, fungos, artrópodes, fezes e sangue, tanto dos filhotes quanto dos adultos (Romoser \& StofFolano 1994). Os artrópodes podem ser alados ou não, com predomínio dos ápteros de pequeno porte, e ter grupos tróficos bastante variados, incluindo espécies detritívoras, hematófagas, parasitóides, predadoras e fitófagas. Os que vivem e se multiplicam em ninhos de aves podem apresentar importância econômica e na saúde pública (DöHring 1958; Popendiker 1959; WeIDNER 1961; TeSChNer 1964; KraAl 1981; KRÜGER 1984; VallvÉ et al. 1995; HAAG-WACKERNAGEL \& SPICWAK, 2004).

Este trabalho realizou um estudo sobre os artrópodes que vivem em ninhos de C. livia com o intuito de inventariar as espécies associadas e verificar quais possuem importância médica ou econômica.

Foram coletados 14 ninhos de $C$. livia em nove bairros de Manaus, Amazonas no período de julho a novembro de 2005. Todos os ninhos foram coletados com a utilização de equipamentos de proteção individual, sendo eles macacão, luvas, óculos e máscaras. A maioria das áreas da cidade foi representada, exceto a região Nordeste e os extremos Norte e Oeste. Os ninhos foram levados ao Laboratório de Triatominae e Phlebotominae, na Coordenação de Pesquisas em Entomologia (CPEN) do Instituto Nacional de Pesquisas da Amazônia (INPA). Foram colocados em um extrator do tipo Berlese-Tullgren por 12 dias, tendo seus frascos coletores trocados a cada dois dias. Após esse período, foi feita uma inspeção para verificar se algum artrópode permanecia no ninho. O material foi fixado em álcool 70\% e depositado na Coleção de Invertebrados do INPA. Para o cálculo da diversidade foi utilizado o índice de Shannon.

Os 14 ninhos coletados foram encontrados sobre cinco tipos de substratos (cimento, ferro, tecido, madeira e terra) e sob dois tipos de coberturas (cerâmica e amianto além de ninhos sem cobertura). A altura do local de coleta variou de o a 20 metros e o peso de cada ninho de 9,85 a 500,03 gramas. Os ninhos que apresentaram o maior número de espécimes foram os que estavam construídos sobre cimento e madeira e em locais cobertos. Ninhos descobertos estão mais expostos a ação do sol e das chuvas, o que pode comprometer a colonização dos ninhos, quanto ao substrato, a maioria dos ninhos estava construída sobre cimento e madeira, que são os principais materiais das residências de Manaus, nos outros substratos foram encontrados apenas um ninho. Foram coletados 10.323 artrópodes (Tabela 1), com uma variação de 5 a 7.541 indivíduos por ninho. A Classe Arachnida foi a mais abundante, sendo Acari a ordem com maior representatividade, variando de o a 6.728 indivíduos por ninho. Hexapoda apresentou a maior diversidade de ordens, sendo Collembola a mais abundante seguida de Psocoptera. Do total de 33 famílias, Acarididae foi a que apresentou maior abundância, tanto entre os ninhos, quanto por ninho. Aranhas da família Ctenidae, com aproximadamente $2 \mathrm{~cm}$, foram os maiores artrópodes encontrados. Em um ninho (ninho 7) foi registrado mais de 95\% de Collembola e Diptera. Formigas do gênero Pheidole Westwood, 1839 corresponderam a $72 \%$ do total 


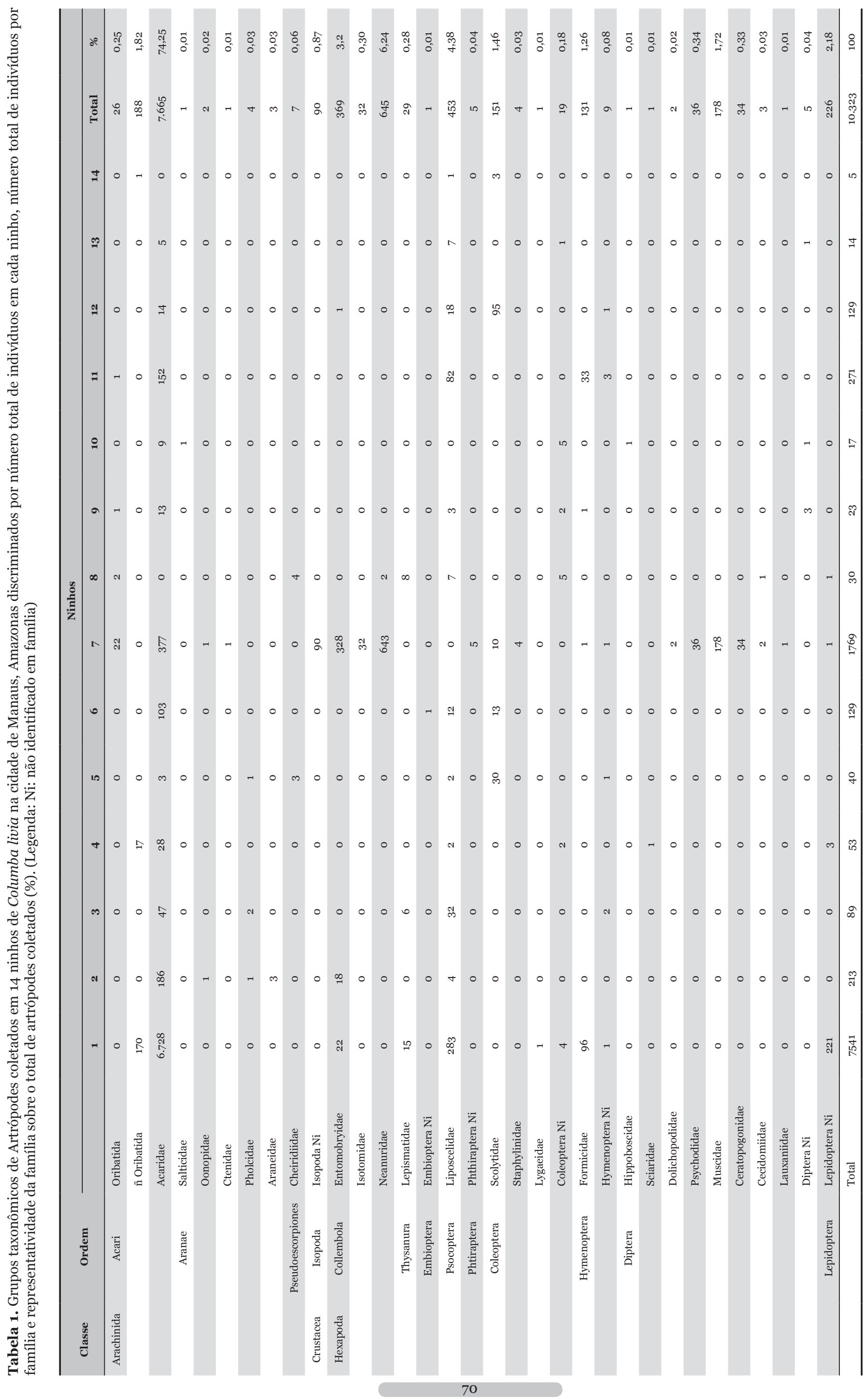


de Formicidae. O índice de Shannon mostrou baixa diversidade para as famílias no total dos ninhos amostrados (H1=1,6941).

Do total de artrópodes coletados $82,26 \%$ podem ser considerados de interesse econômico ou de interesse em saúde pública, sendo estes pertencentes aos grupos Thysanura (Lepisma saccharina L., 1758), Psocoptera (Liposcelis bostrychopila Badonnel, 1931), Acari (Caloglyphus), Diptera (Hippoboscidae) e Hymenoptera (Pheidole, Solenopsis e Brachymyrmex).

A ausência de amostragem na região Nordeste e no extremo Norte e Oeste da cidade é justificada por não terem sido encontrados nem os ninhos e nem as aves de C. livia, e em outros casos pela inacessibilidade ao local do ninho, em local muito alto ou estreito, ou pelo acesso negado pelo proprietário do imóvel. Todos os ninhos coletados foram encontrados em situações parecidas, na maioria em casas, sempre relacionados ao telhado. Um ninho apresentou quase a totalidade de colêmbolos e dípteros encontrados, provavelmente porque esse ninho estava no chão, tendo sido retirado de seu local de construção um dia antes da coleta facilitando o acesso a estes grupos. Em todos os ninhos foram encontrados artrópodes, porém a diversidade neste trabalho é baixa se for comparada a trabalhos realizados com artrópodes nidícolas de aves nativas (Torres 2001; GouveIA 2003). Isso porque o ambiente urbano é muito mais seletivo que o natural, ou seja, nas cidades, os artrópodes encontram uma disponibilidade menor de alimentos e microhabitats favoráveis. Sendo assim, um grupo reduzido (menos diverso) e mais adaptado tende a apresentar uma abundância maior. Também ocorre uma diversidade menor, quando comparada à encontrada em trabalhos com ninhos de pombos realizados na região Paleártica (HAAG-WACKERNAGEL 1998). Isto pode ter ocorrido devido a C. livia ser nativa da região Européia, assim, alguns artrópodes podem ter co-evoluído com os pombos, se adaptando bem as condições existentes em seus ninhos. Já na região Neotropical, os ninhos de aves nativas, que se encontram distribuídos pela área urbana, apresentam melhores condições a artrópodes nativos.

De acordo com os resultados, conclui-se que os artrópodes mais abundantes em ninhos de C. livia na cidade de Manaus são os que possuem característica biogeográfica semelhante à mesma, ou seja, são cosmopolitas e possuem uma ligação direta com o habitat criado por humanos, devido a seu hábito alimentar e aos locais de reprodução.

A introdução desta ave no ambiente urbano pode ter contribuído para um maior contato de alguns artrópodes nidícolas com o ser humano, o que poderá acarretar problemas de cunho econômico ou de saúde pública.

Os indivíduos da espécie Lepisma saccharina Linnaeus, 1758 (Ordem Thysanura), são comuns em residências e conhecidos por atacar livros e tecidos principalmente. É possível que encontrem alimento e abrigo nos ninhos (Borror et al. 1989; Romoser \& STOFFolano 1994).

O psocóptero Liposcelis bostrychophila Badonnel, 1931 é considerado praga por causar prejuízo financeiro, tanto por inviabilizar os grãos, como pelo dinheiro gasto em seu controle (Gallo et al. 2002). Esta espécie é cosmopolita, comum em residências, ambientes silvestres e encontrados também em grãos armazenados (TURNER 1994). L. bostrychophila pode viver um longo período sem alimento e devido a seu pequeno tamanho é altamente invasiva (ALDRETE 1988; BECKETT 2001). A proximidade de ninhos de C. livia com residências pode facilitar o acesso destes insetos aos alimentos estocados em cozinhas (Turner \& MOUdERохву 1989). A essa espécie também está atribuída importância médica por causar reações alérgicas e infestação oportunista em unhas infectadas por micose (Lin et al. 2004).

Os dípteros Hipobboscidae são conhecidos por parasitarem pombos e provocar picadas ocasionais em humanos (HAAG-WACKERNAGEL 1998).

A maioria dos ácaros coletados pertence ao gênero Caloglyphus Berlese, 1923 cujos indivíduos já foram encontrados se alimentando de bulbos de vegetais comercializados (Athanassiou et al. 2002; Sousa et al. 2005) e de alimentos armazenados. Por serem comuns em alimentos estocados, podem carregar fungos patogênicos aos alimentos (Zheltikova et al. 1997; HUBERT et al. 2004). Estes ácaros também podem infestar residências e podem causar reações alérgicas respiratórias e na pele (Solarz et al. 1999; BinotTi et al. 2001; KHOELER 2003). As formigas encontradas (Pheidole, Solenopsis Westwood, 1840 e Brachymyrmex Mayr, 1868) podem ser consideradas de importância social uma vez que são comuns em residências, onde podem fazer ninhos danificando estruturas e infestando alimentos (Robinson 1996). De acordo com o local de construção do ninho, todos estes artrópodes podem invadir residências, e causar os danos descritos (HAAG-WACKERNAGEL \& SPICWAK 2004). $\mathrm{Na}$ verdade, a simples presença de qualquer artrópode dentro de uma residência pode ser considerada prejudicial. O prejuízo neste caso não é financeiro ou à saúde, é psicológico pela presença de um "invasor" na residência (ROBINSON 1996; HAAG-WACKERNAGEL \& SPICWAK 2004).

\section{REFERÊNCIAS}

Aldrete, A.N.G., 1988. Especies de Psocidos (Psocoptera) en Nidos de Aves en Mexico. Anales del Instituto Biologico serie Zologia, 58: 507-524.

Athanassiou, C.G., N.E. Palyvos, P.A. Eliopoulos \& G. T. Papadoulis, 2002. Mites associated with stored seed cotton and related products in Greece. Phytoparasitica, 30: 387394 .

Beckett, S., 2001. Treating psocids with heat: an alternative grain disinfestations treatment for a new pest. Stored Grain Laboratory, CSIRO Entomology ACT 2601, Camberra.

Binotti, R.S., J.R.O. Miniz, I.A. Paschoal, A.P. Prado \& C.H. Oliveira, 2001. House dust mites in Brasil- an anoted bibliography. Memórias do Instituto Oswaldo Cruz, 96: 11771184 .

Borror, D.J., C.A. Triplehorn \& N.F. Johnson. 1989. Study of Insects. Sanders College Publishing $809 \mathrm{pp}$.

Chiappe, L.M. \& G.J. Dyke, 2002. The Mesozoic radiation of birds. Annual Review of Ecology and Statistic, 33: 91-124.

Döhring, V.E., 1958. Plagen durch verwilderte Haustauben. Ornitologische Mitteilungen, 3: 163-168.

Gallo, D., O. Nakano, S.S. Neto, R.P.L Carvalho, G.C. Baptista, E.B. Filho, J.R.P. Parra, R.A. Zucchi, S.B. Alves, J.D. Vendramin, L.C. Marchini, J.R.S. Lopes \& C. Omoto, 2002. Entomologia Agrícola. FEALQ Piracicaba. Vol 10. p 920.

Gouveia, F.B.P., 2003. Artrópodes associados a ninhos de Cacicus sp. e Psarocolius sp. (Passeriformes: Emberezidae: Icterinae) em áreas de várzea próximas á confluência dos rios Negro e Solimões, no período de cheia, na Amazônia central, Brasil. Dissertação de Mestrado Instituto Nacional de Pesquisas da Amazônia/Fundação Universidade do Amazonas, Manaus, Amazonas 123 pp.

Haag-Wackernagel, D., 1998. Die Taube. Vom heiligen Vogel der Liebesgöttin zur Strassentaube. Schwabe \& Co. AG, Basel, 245p.

Haag-Wackernagel, D. \& C. Spicwak, 2004. Human infestation by pigeon fleas (Ceratophyllus columbae) from feral pigeons. Annals of Agriculture, Enviroment and Medicine, 11: 343356.

Hicks, E.A., 1959. Checklist and bibliography of the occurrence of insects in bird's nests. Ames, Iowa. Iowa State College Press. 681p.

Hubert, J., V Stejskal, Z. Munzbergova A. Kubatova, M. Vanova \& E. Zd'arkova, 2004. Mites and fungi in heavily infested stores in the Czech Republic. Journal of Economical Entomology 97: 2144-2153.

Koehler, P.G., 2003. Mites that attack Humans. University of Florida Extensions 6: 1-6.

Kraal, S., 1981. Ökofaunistische Untersuchugen der Insekten in Nestern der Stadttaube (Columba livia domestica L.) unter besonderer Berücksichtigung schädlicher und Arten. Entomologische Mittelugen. Zoologische Museum, 7: 29-44. Krüger, H.V., 1984. Die Nestfauna verwilderter Haustauben 
und ihre gesundheitsschädigenden Spezies. Monatash Veterinaire, 39: 241-246.

Lin, Y.C., M.L. Chan C.W. Ko \& M.Y. Hsieh, 2004. Concise report Nail infestation by Liposcelis bostrychophila Badonnel. Clinical and Experimental Dermatology 29: 620.

New, T.R., 1972. Some Brazilian Psocoptera from bird nest. The Entomologist, 105: 153-160.

Popendiker, K., 1959. Die in Vogelnestern lebenden Insekten unter besonderer Berücksichtigung der als haus und Vorratsschädlinge auftretenden Arten. Mittelugem die Hamburg Zoologische Museum and Institut, 5: 54-59.

Robinson, H.W., 1996. Urban Entomology, Insect and mite pest in the human environment. 1th ed., Ed. Chapman \& Hall 432 p.

Romoser, W.S. \& J.G. Stoffolano, 1994. The Science of Entomology. 3d Edition. WCB McGraw-Hill, Dubuque, Iowa. $605 p$.

Solarz, K., O. Szilman \& E. Szilman, 1999. Allergenis mites associated withbirdnestsin Poland(Astigmata, Pyroglyphidae, Acaridae, Glycyphagidae). p.651-656. In Bruin J, van der Geest LPS, Sabelis MW: Ecology and Evolution of the Acari. Kluwer Academic Publishers, Dordrech. 692 pp.

Sousa, J.M., M.G.C. Gondim, R. Barros \& J.V. Oliveira, 2005. Ácaros em Produtos Armazenados Comercializados em Supermercados e feiras Livres da Cidade do Recife. Neotropical Entomology 34: 303-309.

Teschner, V.D., 1964. Die Bedeutung der Nester verwilderter Tauben in Großstädten. Zoologische Institut Staatl Brauschweig, Natur Museum, 5: 40-43.

Torres, W.M P., 2001. Fauna de artrópodes associada a ninhos de aves e mamíferos e uma floresta de terra firme na Amazônia

Como citar este artigo:

Ogawa, G.M., 2008. Artrópodes em ninhos de Columba livia Gmelin, 1789 (Aves, Columbidae) em área urbana de Manaus, Amazonas, Brasil. EntomoBrasilis, 1(3): 69-72. www.periodico.ebras.bio.br/ojs
Central, Brasil. Dissertação de mestrado em Entomologia Instituto Nacional de Pesquisas da Amazônia/Fundação Universidade do Amazonas, Manaus, Amazonas, 243 pp.

Turner, B.D., 1994. Liposcelis bostrychophila (Psocoptera; Liposcelididae), a stored product food pest in the UK. International Journal of Pest Management 40: 179-190.

Turner, B.D. \& H. Maude-Roxby, 1989. The prevalence of the booklouse Liposcelis bostrychophila Badonnel (Liposcelidae, Psocoptera) in British domestic kitchens. International Pest Control, 31: 93-97.

Vallvé, S.L., H. Rojo, \& C. Colli-Wisniesky, 1995. Ecologia Urbana de Triatoma infestans em Argentina. Asociación entre Triatoma infestans y palomares. Revista de Saúde Pública., 29: 192-198.

Weidner, H., 1961. Die Niststätten verwilderte Tauben als reservoire für Ungezieferplagen. Städtehygiene, 5: 91-94.

Wlodarczyk, J., 1963. Psocoptera of some bird nests. Fragmenta Faunística, 10: 361-366.

Zheltikova, T.M., V.B. Gervazieva, S.N. Zhirova, M.A. Mokronosova \& V.V. Sveranovskaia, 1997. Storage mites as the source of household allergens. Zhournal Mikrobiologi Epidemiologi Immunobiologi 6: 73-76.

Recebido em: 05/06/2008

Aceito em: 15/09/2008

$* * * * * * * * * * * * *$

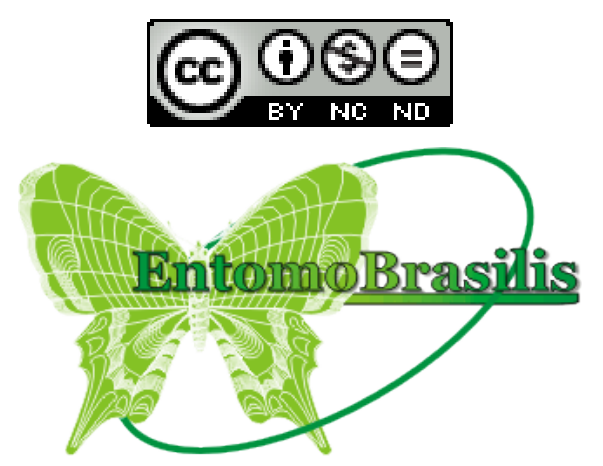

